\title{
EL ESTILO PERSONAL DEL TERAPEUTA EN PROFESIONALES CHILENOS E ITALIANOS
}

\section{THE PERSONAL STYLE OF THE THERAPIST IN CHILEAN AND ITALIAN PROFESSIONALS}

\author{
Álvaro Quiñones', Carla Ugarte², Francisco Ceric ${ }^{3}$, Paola Cimbolli ${ }^{4}$, \\ Fernando García ${ }^{5}$ y Adele De Pascale ${ }^{6}$ \\ 1 Profesor Asociado. Facultad de Psicología, Universidad San Sebastián, Santiago, Chile \\ ORCID: https://orcid.org/0000-0002-6993-0324 \\ 2 Profesor Asistente. Facultad de Psicología, Universidad San Sebastián, Santiago, Chile \\ ORCID: https://orcid.org/0000-0002-8130-131X
}

3 Profesor Asociado. Centro de Apego y Regulación Emocional (CARE), Facultad de Psicología, Universidad del Desarrollo, Santiago, Chile ORCID: https://orcid.org/0000-0002-5526-3399

4 Centro Clinico Nemo Roma, Fondazione Policlinico Universitario Agostino Gemelli

IRCCS, Italia

ORCID: https://orcid.org/0000-0002-2328-7900

5 Investigador. Fundación Aigle, Buenos Aires, Argentina

6 Director of APR, Accademy of Post-Rationalist Psychotherapy, Psychiatry and Cultural

Psychology. Roma, Italia

ORCID: https://orcid.org/0000-0001-8172-0111

Cómo referenciar este artículo/How to reference this article:

Quiñones, A., Ugarte, C., Ceric, F., Cimbolli, P., García, F. y De Pascale, A. (2020). El Estilo Personal del Terapeuta en Profesionales Chilenos e Italianos. Revista de Psicoterapia, 31(116), 191-203. https://doi.org/10.33898/rdp.v31i116.345

\begin{abstract}
Resumen
El estilo personal del terapeuta (PST) es una construcción multidimensional que está presente en cada proceso terapéutico. Objetivo. Estudiar las diferencias entre los terapeutas cognitivos post-racionalistas italianos y chilenos en el estilo personal del terapeuta. Método. Se realizó un análisis transversal para comparar terapeutas en las cinco dimensiones de EPT. Para este propósito, se aplicó una prueba t. El cuestionario EPT-C se administró a una muestra total de 63 terapeutas cognitivos post-racionalistas de ambas nacionalidades. Resultados. Se encontraron diferencias estadísticamente significativas entre la edad de los terapeutas en las funciones expresiva $(p<0.05)$ e involucración $(p<0.05)$. Los terapeutas italianos mostraron una media significativamente mayor en la función expresiva. Conclusión. En las cinco funciones evaluadas por ETP solo se encontraron diferencias significativas para la función expresiva, cuando se comparó por nacionalidad.

Palabras clave: Estilo personal del terapeuta, psicoterapia Cognitiva Postracionalista, investigación, cultura.
\end{abstract}

Fecha de recepción v1: 31-1-2020. Fecha de recepción: v2: 15-3-2020. Fecha de aceptación: 25-4-2020. Correspondencia sobre este artículo:

E-mail: alvaroquinonesb@gmail.com

Dirección postal: Álvaro Quiñones. Instituto de Ciencias Humanas Aplicadas (ICHA), Facultad de Psicología, Universidad San Sebastián, Santiago, Chile 


\section{Abstract}

The Personal Style of the Therapist (PST) is a multidimensional construction that is present in every therapeutic process. Aim. Study the differences between Italian and Chilean cognitive post-rationalist therapists in the therapist's personal style. Method. A cross-sectional analysis was conducted to compare therapists in the five dimensions of EPT. For this purpose, a t-test was applied. The EPT-C questionnaire was administered to a total sample of 63 cognitive post-rationalist therapists of both nationalities. Results. Statistically significant differences were found between the age of the therapists in the expressive $(p<0.05)$ and involvement $(p<0.05)$ functions. Conclusion. In the five functions evaluated by ETP only significant differences were found for the expressive function, when compared by nationality.

Keywords: Personal style of the therapist, Cognitive post-rationalist psychotherapy, research, culture. 


\section{Introducción}

En la actualidad contamos con investigación y hallazgos significativos sobre las variables que participan en el proceso y el resultado de la psicoterapia. Conjunto de conocimiento que se ha logrado a través de diversos estudios: focalizados en el terapeuta, en el paciente, y en la relación terapéutica (Beutler et al., 2004; García y Fernández-Alvarez, 2007; Orlinsky, Grawe y Parks, 1994; Orlinsky y Howard, 1986; Quiñones, Ceric, Ugarte y De Pascale, 2017; Quiñones, Ceric y Ugarte, 2015; Quiñones, Melipillán y Ugarte, 2012; Quiñones, Ugarte, Ceric, García y Santibáñez, 2019; Wampold, 2015; Wampold e Imel, 2015).

Es importante comprender y asumir que la psicoterapia se lleva adelante en una cultura determinada. La cultura se refiere a las formas comunes en que los individuos interpretan el significado de ellos mismos y sus mundos posibles (Bruner, 1988, 1991; La Roche y Christopher, 2009). Los miembros de la misma cultura comparten conocimientos, música, valores, creencias, costumbres, rituales e historia, entre otras cosas. Toda esa dinámica de elementos culturales crea un sentido compartido de identidad y significados comunes y públicos. Más específicamente, Marsella y Yamada (2010), en su esfuerzo por discernir el vínculo entre cultura y comportamiento humano, definieron la cultura de la siguiente manera:

La cultura es el comportamiento y los significados aprendidos que se transmiten socialmente con fines de ajuste y adaptación. La cultura está representada externamente en artefactos (por ejemplo, comida, ropa, música), roles (por ejemplo, la formación social) e instituciones (por ejemplo, familia, gobierno). Está representado internamente (es decir, cognitivamente, emocionalmente) por valores, actitudes, creencias, epistemologías, cosmologías, patrones de conciencia y nociones de personalidad. La cultura se codifica verbalmente, imaginariamente, propioceptivamente, visceralmente y emocionalmente, dando como resultado diferentes estructuras y procesos experienciales (2010, p.105, Traducción libre de los autores).

Sumado a lo anterior, es fundamental también destacar que el contenido de la cognición es siempre intersubjetivo y, en tal sentido, la sensibilidad cultural del Homo Sapiens es de la mayor relevancia en el significado expresado principalmente a través de las narrativas y del estar con otros en la construcción del significado (Quiñones, 2013). A modo de ejemplo de cómo la cultura incide en el procesamiento de la información y del significado, Cai, Brown, Deng y Oakes (2007), destacan que existe un consenso sobre la naturaleza de las diferencias culturales en la autoestima; los autores indican los siguientes hechos: (a) las personas de todo el mundo se esfuerzan por sentirse bien con ellas mismas; (b) las culturas dictan qué cualidades deben tener las personas para sentirse bien con ellas mismas; (c) los asiáticos obtienen puntuaciones más bajas en las escalas de autoestima que los occidentales; (d) estas diferencias surgen porque los asiáticos evalúan sus características de manera menos positiva, no porque se gusten menos que los occidentales; y (e) las diferencias culturales en las autoevaluaciones dependen, al menos en 
parte, de las diferencias culturales en las normas de modestia. En tal contexto de diferencias culturales, nos interesamos en el estudio del terapeuta y en cómo las eventuales diferencias pueden afectar su estilo personal de trabajo en psicoterapia.

El foco de nuestra investigación es el estilo personal del terapeuta. Es un constructo multidimensional y es un componente de todo proceso terapéutico (Fernández-Álvarez, García, Lo Bianco y Corbella, 2003; Fernández-Álvarez, García y Schreb, 1998). El constructo fue definido por Héctor Fernández-Álvarez et al., (1998):

El estilo personal del terapeuta (PST) se ha definido como un conjunto de condiciones singulares que llevan a un terapeuta a operar de una manera particular en su trabajo (Fernández-Alvarez, en prensa). Esto se refiere a las características normales que cada terapeuta imprime en su trabajo como resultado de su peculiar forma de ser, independientemente del enfoque de trabajo que utiliza y los requisitos específicos que exige su intervención (1998, p. 353, Traducción libre de los autores).

Hoy por hoy, hay cerca de 48 publicaciones que han utilizado el cuestionario EPT-C (Fernández Álvarez y García, 2019). Por otra parte y según orientaciones, las investigaciones muestran lo siguiente:

a) Los terapeutas psicoanalistas presentan menores puntuaciones en las dimensiones Atencional y Operativa (atención abierta y tratamientos menos pautados) y puntuaciones bajas en la dimensión expresiva (menos cercanos) (Fernández-Álvarez, García, Lo Bianco y Corbella, 2000) e involucramiento (Casari, Albanesi y Maristany, 2013; Castañeiras, Ledesma, García y Fernández-Álvarez, 2008; Fernández-Álvarez et al., 2000; Quiñones y Ugarte, 2019).

b) Los terapeutas cognitivos (racionalistas), serían más directivos en su estilo y más distantes emocionalmente (Vides-Porras, Grazioso y García de la Cadena, 2012). Muestra de 101 terapeutas, en que un 13.9\% se identificó como Cognitivo y un $7.9 \%$ se identificó como Cognitivo-Humanista. No se incluyen terapeutas de orientación post-racionalista.

c) Los terapeutas sistémicos presentan mayores puntuaciones en el estilo involucración y preferencia por tratamientos menos pautados(Vides-Porras et al., 2012).

d) En una muestra de 60 terapeutas de tres orientaciones: 20 psicoanalistas, 20 cognitivos y 20 integrativos. No obstante, lo pequeño del tamaño muestral de los grupos, reportaron un perfil con diferencias significativas, y por tanto característico, según los terapeutas sean cognitivos, integrativos o psicoanalíticos (Vázquez y Gütierrez de Vázquez, 2015).

e) En una muestra de 67 terapeutas se comparó a la orientación cognitiva post-racionalista y sistémica (Quiñones et al., 2019; Quiñones y Ugarte, 2019). Se realizó una prueba $t$ de comparación de medias (bilateral) para determinar si existían diferencias significativas en las puntuaciones de 
ambos grupos en las distintas funciones del EPT-C. El análisis mostró que los terapeutas sistémicos y cognitivos post-racionalistas presentan diferencias estadísticamente significativas en dos funciones. Función atencional ( $t=-2.541 ; p<0.05$ y un tamaño del efecto $r=0.296)$ y función operativa ( $t=-2.253$; $p<0.05 \mathrm{y}$ un tamaño del efecto $r=0.267$ ). En ambos casos se evidencia un tamaño de efecto pequeño. En las demás funciones se observan diferencias leves en los puntajes que no alcanzan a ser significativas.

En tal contexto, es de destacar que el cuestionario EPT-C se encuentra validado en diferentes idiomas, lo que muestra buena sensibilidad del instrumento psicométrico (Da Silva, Tellet, Fernández-Álvarez y García, 2006; García y Fernández-Álvarez, 2007; Lee, Neymar y Rice, 2013; Prado-Abril et al., 2019; Vides-Porra et al., 2012). Sumado a lo anterior, encotramos únicamente una referencia en que se aborda una comparación multicultural con el EPT-C presentada en un congreso y que hasta la fecha no ha sido publicada (García, Castañeiras, Rial y Fernández-Álvarez, 2005).

En el presente estudio se evaluará las diferencias entre las funciones del ETP-C entre terapeutas chilenos e italianos. Lo anterior, se fundamenta en que la expresión emocional está situada en un encuadre cultural. La expresión emocional en Italia es diferente a la expresión de emociones en Chile. Si bien es cierto, según nuestra búsqueda en bases de datos, no hay estudios que muestren una comparación de la expresión emocional entre chilenos e italianos. En nuestra opinión, la diferencia posible tiene que ver con que la dimension expresiva del EPT-C debiera reflejar esta diferencia.

El objetivo de la presente investigación fue evaluar si existen diferencias en las funciones del Estilo Personal de Terapeuta (EPT-C), según sexo, edad y nacionalidad de los terapeutas Cognitivos Post-racionalistas chilenos e italianos.

La hipótesis principal de investigación que condujo el presente trabajo fue que existen diferencias en la función expresiva del Estilo Personal del Terapeuta.

En último lugar, es de destacar, que la presente investigación según lo revisado en distintas bases de datos (WoS, ScieLo, PsicoInfo, MEDLINE, EBSCO), es el segundo estudio que investiga a terapeutas de la escuela cognitivo post-racionalista en el constructo Estilo Personal del Terapeuta, utilizando el cuestionario EPT. Es de destacar, que el primer estudio publicado es una compraración de dos escuelas, terapeutas post-racionalistas y terapeutas sistémicos (Quiñones et al., 2019). De igual manera, este estudio es el primero que compara una misma escuela psicoterapéutica en dos paises con idiomas distintos: Italia y Chile.

\section{Método}

Para este estudio se realizó un análisis comparativo de alcance descriptivocorrelacional, a través de un diseño no experimental y de corte transversal. El muestreo fue no probabilístico de tipo intencionado.

Los participantes fueron seleccionados en base a los siguientes criterios de 
inclusión: a) Psicólogos que ejercen psicoterapia; b) que tengan formación y que se autodenominen perteneciendo a la orientación Cognitivo Post-racionalista.

\section{Instrumentos}

El constructo Estilo Personal del Terapeuta fue evaluado con la versión del cuestionario de Evaluación del Estilo Personal del Terapeuta -EPT-C- (FernándezÁlvarez y García, 1998; Fernández-Álvarez et al., 2003) validado en Chile por Quiñones, Melipillán y Ramírez (2010). El EPT-C es un instrumento autoadministrado consta de 36 ítems presentados como aseveraciones a las que cada terapeuta debe responder según su grado de acuerdo en una escala de tipo Likert, que va desde 1 (totalmente en desacuerdo) a 7 (totalmente de acuerdo). Los 36 ítems se agrupan en las siguientes 5 funciones:

a) Función instruccional [flexibilidad-rigidez]: Alude al establecimiento del encuadre terapéutico y la actitud que toma el profesional con respecto a los limites.

b) Función expresiva [distancia-cercanía]: Alude a la expresividad emocional. Muestra la intensidad emocional de los intercambios emocionales que ocurren en el proceso psicoterapéutico.

c) Función de involucración [menor grado-mayor grado]: Alude al grado de compromiso que el psicoterapeuta experimenta con su trabajo y la relación con el resto de las áreas vitales.

d) Función atencional [amplio-focalizado]: Relacionada con la búsqueda de información que puede ser focalizada o abierta.

e) Función operativa [espontáneo- pautado]: Se refiere a las acciones que realiza el terapeuta en relación a las intervenciones terapéuticas específicas. Permite diferenciar profesionales con actitud directiva versus actitud espontánea en relación a las intervenciones.

El cuestionario ha presentado una adecuada confiabilidad y estabilidad temporal (test-retest a 4 meses) para las 5 funciones (Fernández-Álvarez et al., 2003).

Específicamente, en cuanto a la fiabilidad la versión original creada en Argentina (Fernández-Álvarez et al., 2003) presentó adecuados niveles, con valores alfa de Cronbach de entre .69 (función instruccional) a .80 (función atencional). En terapeutas chilenos los valores de fiabilidad según alfa de Cronbach fueron iguales o mayores a .75, con excepción de la función de involucración que obtuvo un alfa de .68 (Quiñones et al., 2010). Finalmente, en terapeutas italianos, la fiabilidad osciló entre los valores .61 (función atencional) a .74 (función expresiva) (Cimbolli, Quiñones, Ugarte y Ceric, 2017).

Cuestionario sociodemográfico y de formación profesional: Se construyó un cuestionario ad hoc para la presente investigación, tanto en español como en italiano, a través del que se consignaron datos como la edad, sexo, años de experiencia, grado académico y enfoque terapéutico, contexto de ejercicio profesional y acreditación como psicólogo clínico. 


\section{Procedimiento}

En primer lugar se hizo una planilla con los nombres de los terapeutas conocidos por los investigadores chilenos e italianos, que tenían formación en el enfoque Cognitivo Post-racionalista. En segundo lugar, se envió una invitación en castellano e Italiano a través de correos electrónicos a los psicoterapeutas en Chile e Italia. En el correo en castellano e Italiano, se les describió la investigación y se les facilitó el link que contenía el consentimiento informado, un "cuestionario sociodemográfico y de formación profesional" y el cuestionario EPT-C validado en Chile (Quiñones, et al., 2010) y adaptado en Italia (Cimbolli et al., 2017). Las respuestas evacuadas en el formulario en-linea fueron recepcionadas automáticamente en una planilla excel. Una vez recibidas las respuestas de los terapeutas, la planilla excel fue traspasada al software estadístico SPSS v.21 para proceder a su depuración y análisis estadístico.

\section{Plan de análisis}

Primero, se realizaron los análisis descriptivos del instrumento y la muestra en estudio. Además, se analizó la consistencia interna de las funciones del ETP-C para esta muestra. Las variables fueron descritas según normalidad. Posteriormente, se utilizó la prueba $t$ de Student para analizar la relación entre sexo y las funciones del EPT-C. Para analizar la relación entre la edad de los terapeutas y las funciones de EPT-C se aplicó correlación de Pearson, y finalmente, para estudiar la relación entre la nacionalidad (aspecto cultural) y las funciones del EPT-C se realizó la prueba $t$ de Student.

\section{Resultados}

\section{Estadísticos Descriptivos}

La muestra estuvo conformada por un total de 63 terapeutas, de los cuales un $62 \%(n=39)$ eran mujeres y $38 \%(n=24)$ hombres. El promedio de edad fue de de 44 años $(D T=11.09)$, con un rango de edad entre 27 y 72 años. El 54\% de los terapeutas eran chilenos $(n=34)$.

Los 63 terapeutas, son de orientación cognitiva post-racionalista. En cuanto al nivel de experiencia, la media de años de ejercicio profesional en el grupo de terapeutas post-racionalistas chilenos fue de 12.01 años $(D T=9.98)$ y de 14.93 años $(D T=10.78)$ en los terapeutas italianos.

El 67.6\% $(n=23)$ del grupo de terapeutas de orientación cognitiva postracionalista chileno ejerce mayormente en la atención privada y el resto en el sector público. Para el grupo de terapeutas post-racionalistas italianos, se evidencia que el 72.4\% $(n=21)$ lo hace en atención privada (ver Tabla 1$)$. 
Tabla 1. Descriptivos de la muestra.

\begin{tabular}{cccccccc}
\hline Grupo & $n$ & $\begin{array}{c}\text { Edad } \\
M(D T)\end{array}$ & $\begin{array}{c}\text { Sexo } \\
\text { (\% femenino) }\end{array}$ & $\begin{array}{c}\text { Años Ejercicio } \\
M(D T)\end{array}$ & $\begin{array}{c}\text { Con formación } \\
\text { Post-racionalista }\end{array}$ & $\begin{array}{c}\text { Atención } \\
\text { (\% privada) }\end{array}$ & $\begin{array}{c}\text { Habilitado } \\
\%\end{array}$ \\
\hline Italianos & 29 & $\begin{array}{c}47.55 \\
(10.37)\end{array}$ & $76 \%$ & $14.93(10.78)$ & $100 \%$ & $72.4 \%$ & $65 \%$ \\
Chilenos & 34 & $\begin{array}{c}42.98 \\
(11.85)\end{array}$ & $50 \%$ & $12.01(9.98)$ & $100 \%$ & $67.6 \%$ & $24 \%$ \\
\hline
\end{tabular}

La fiabilidad del instrumento para la presente muestra, se evaluó mediante alfa de Cronbach, obteniéndose alfa de .613 para la función atencional, .646 para la función expresiva, .669 para la función instruccional, .626 para la función involucración, y .636 para la función operativa.

Los estadísticos del instrumento, se presentan en la Tabla 2.

Tabla 2. Descriptivos del instrumento.

\begin{tabular}{lcccc}
\hline Función EPT-C & Min. & Max. & $M$ & $D T$ \\
\hline Instruccional & 18 & 48 & 32.57 & 6.70 \\
Atencional & 6 & 27 & 18.01 & 4.32 \\
Expresiva & 23 & 63 & 42.65 & 9.07 \\
Involucracional & 13 & 40 & 26.14 & 6.10 \\
Operativa & 8 & 40 & 22.41 & 6.54 \\
\hline
\end{tabular}

\section{Variables sociodemográficas y funciones del ETP-C}

Se evaluó si existían diferencias significativas en las funciones del ETP-C según sexo, ello se utilizó la prueba $t$ de Student. Los resultados no muestran diferencias significativas en las cinco funciones del ETP-C por sexo (véase Tabla 3).

Tabla 3. Prueba t de Student según sexo de los terapeutas post-racionalistas.

\begin{tabular}{lccc}
\hline Función EPT-C & $\begin{array}{c}\text { Hombres } \\
M(D T)\end{array}$ & $\begin{array}{c}\text { Mujeres } \\
M(D T)\end{array}$ & $t(p)$ \\
\hline Instruccional & $31.70(7.5)$ & $33.10(6.1)$ & $-.799(.42)$ \\
\hline Atencional & $18.41(5.3)$ & $17.76(3.5)$ & $.522(.60)$ \\
\hline Expresiva & $43.54(9.1)$ & $42.10(9.0)$ & $.608(.54)$ \\
\hline Involucracional & $26.04(6.8)$ & $26.20(5.6)$ & $-.102(.91)$ \\
\hline Operativa & $23.37(6.8)$ & $21.82(6.3)$ & $.914(.36)$ \\
\hline
\end{tabular}

Por otra parte, se evaluó la relación entre las funciones del ETP-C y la edad de los terapeutas, para ello, se aplicó la correlación de Pearson. Los análisis mostraron que la edad se asoció de forma directa y significativa a la función expresiva $(r=.26 ; p=.040)$ y de forma inversa y significativa a la función instruccional $(r$ $=-.289 ; p<.022)$ (véase Tabla 4$)$. 
Tabla 4. Correlaciones entre edad y funciones del ETP-C.

\begin{tabular}{lllllll}
\hline Variables & 1 & 2 & 3 & 4 & 5 & 6 \\
\hline 1. Edad & 1 & & & & & \\
2. Función instruccional & $-.289^{\star}$ & 1 & & & & \\
3. Función Atencional & .061 & $.259^{\star}$ & 1 & & & \\
4. Función Expresiva & $.260^{\star}$ & $-.252^{\star}$ & -.170 & 1 & & \\
5. Función Involucracional & -.022 & -.126 & -.150 & $.448^{\star *}$ & 1 & \\
6. Función Operativa & -.150 & $.319^{\star}$ & $.465^{\star *}$ & -.190 & $-.271^{*}$ & 1 \\
\hline
\end{tabular}

Nota: ${ }^{*} \mathrm{p}<0,05 ;{ }^{* *} \mathrm{p}<0,01$.

\section{Comparación de grupos según las cinco funciones constituyentes del EPT-C}

Se realizó una prueba $t$ de Student para comparar de medias y determinar si existen diferencias significativas en las puntuaciones de ambos grupos en las distintas funciones del EPT-C (ver Tabla 5 y Gráfico 1). Este análisis evidenció diferencias significativas, solo para la función Expresiva $(t=-3.02$ y $p=.004)$, donde los terapeutas italianos presentan un promedio superior al chileno $(M=$ 46.17 vs. $M=39.64)$.

Tabla 5. Prueba t de Student según nacionalidad de los terapeutas post-racionalistas.

\begin{tabular}{lcccc}
\hline Variable & Terapeutas Italianos & $\begin{array}{c}\text { Terapeutas Chilenos } \\
M(D T)\end{array}$ & $t(p)$ & $d(r)$ \\
\hline Instruccional & $31.5(4.22)$ & $33.4(8.21)$ & $-1.21(.25)$ & - \\
Atencional & $17.3(2.39)$ & $18.5(5.44)$ & $1.14(.26)$ & - \\
Expresiva & $46.1(7.72)$ & $39.6(9.16)$ & $-3.02(.004)$ & $.7(.35)$ \\
Involucracional & $27.7(5.39)$ & $24.7(6.42)$ & $-1.94(.057)$ & - \\
Operativa & $21.2(4.47)$ & $23.3(7.83)$ & $1.33(.18)$ & - \\
\hline
\end{tabular}

Por otra parte, se destaca que la función involucracional mostró una diferencia cercana a la significancia estadística entre los grupos.

En el gráfico 1 se presentan las diferencias de medias en las funciones: instruccional, atencional, expresiva, involucracional y operativa. 


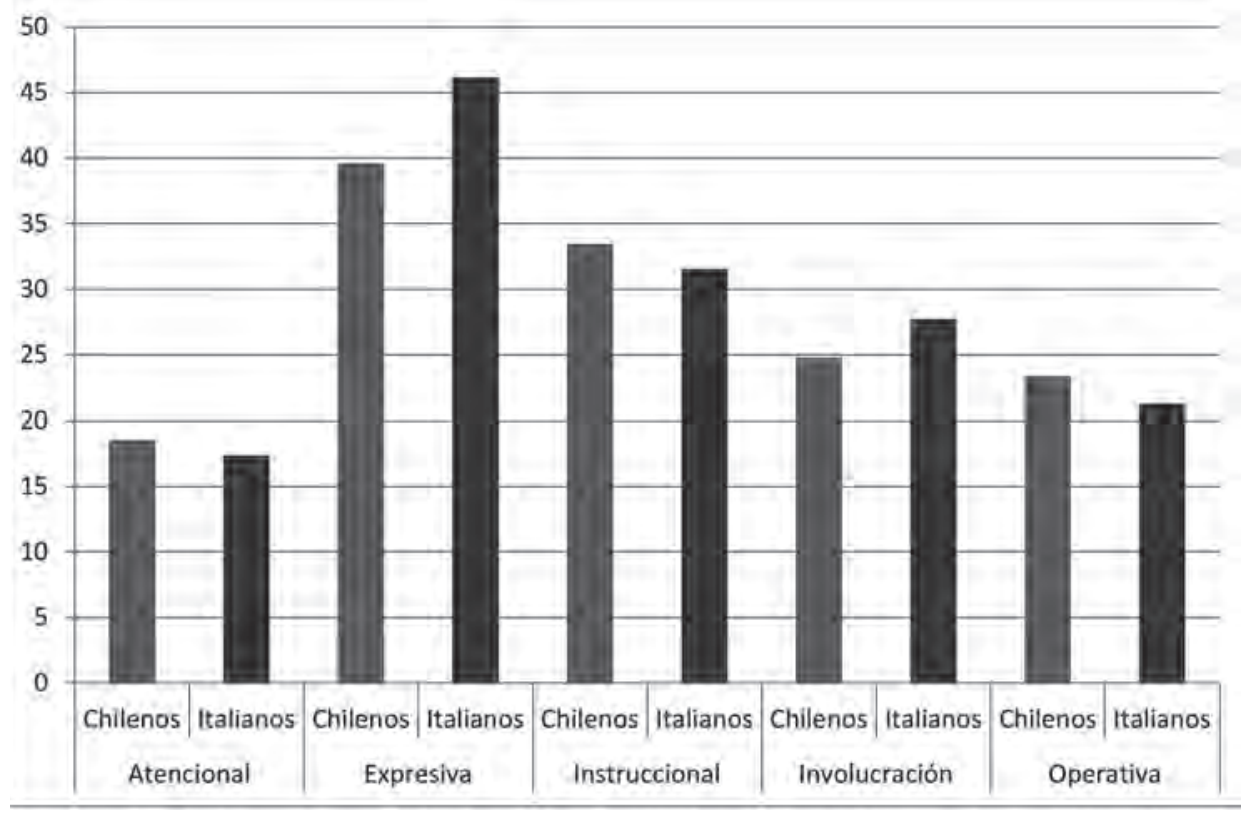

Gráfico 1. Promedios por función del EPT-C.

\section{Discusión}

El objetivo general de investigación fue determinar si existen diferencias en las funciones del Estilo Personal de Terapeuta (EPT) en función del sexo, la edad y la nacionalidad de psicoterapeutas Cognitivos Post-racionalistas chilenos e italianos.

Con respecto a las diferencias en las funciones del EPT-C según sexo, nuestros resultados no muestran diferencias significativas en las cinco funciones del ETP-C. Es de destacar, que nuestros resultados no son coincidentes con lo reportado por Castañeiras et al. (2008), quienes encontraron que los terapeutas hombres presentan una atención más focalizada (función atencional).

En cuanto a las diferencias en las funciones del EPT-C según la edad de los terapeutas, nuestros resultados muestran que la edad se asoció de forma directa y significativa a la función expresiva $(r=.26 ; p=.040)$ y de forma inversa y significativa a la función instruccional $(r=-.289 ; p<.022)$. Estos resultados no son coincidentes con lo reportado en Castañeiras et al. (2008) puesto que ellos reportaron que la edad estaba relacionada a menor de las funciones atencional y operativa.

En relación a las diferencias en las funciones del EPT-C y la nacionalidad de los terapeutas, nuestros resultados muestran diferencias significativas únicamente para la función Expresiva $(t=3.02 ; p=.004)$, donde los terapeutas Italianos presentaron un promedio superior al Chileno. Dicho resultado confirma la hipótesis de investigación sobre que existen diferencias en la función expresiva del Estilo Personal del Terapeuta. 
También nuestra investigación señala la importancia de considerar la variable cultural. En particular, apoya que la psicoterapia se lleva adelante en una cultura determinada y debe incorporar una sensibilidad y perspectiva de diferencias culturales en el procesamiento de información y construcción de significado. Por lo que nuestra investigación evidencia que las diferencias culturales pueden afectar el estilo personal del terapeuta. En este sentido, los resultados nos muestran cómo se comporta el constructo en dos muestras de terapeutas que adscriben a una misma escuela de formación. Si bien es cierto, esto no es generalizable, nuestros resultados tienen un valor teórico que otorga una renovada perspectiva para analizar cómo la cultura se implica en el ejercicio de la psicoterapia.

Una primera limitación a considerar de la presente investigación, es que la composición de la muestra es en un $62 \%$ femenina. En la actualidad sabemos que el procesamiento emocional y la diferencia de género debe ser considerado ya que es un tema que sigue abierto a la investigación y debate a la luz de evidencia (Staczan et al., 2017).

Una segunda limitación a considerar, es el tamaño de la muestra y el tipo de muestreo. Esto último implica que nuestros resultados se pueden aplicar solo a la muestra analizada y no pueden ser generalizables.

Y por último, en cuanto a las líneas de investigación a futuro, se visualiza como relevante continuar realizando comparaciones del constructo Estilo Personal del Terapeuta en distintas culturas, junto con la evaluación de otros constructos que puedan estar moderando las posibles diferencias, como por ejemplo rasgos de personalidad.

\section{Conflictos de intereses}

Los autores declaran que no existen conflictos de intereses.

\section{Agradecimientos}

A los terapeutas italianos y chilenos que colaboraron voluntariamente en la presente investigación.

\section{Referencias Bibliográficas}

Beutler, L. E., Malik, M., Alimohamed, S., Harwood, T.M., Talebi, H., ... Wong, E. (2004). Therapist Variables. En M. J. Lambert (Ed.), Bergin and Garfield's Handbook of Psychotherapy and Behavior Change. Fifth Edition (pp. 227-306). Nueva York, NY: Wiley.

Bruner, J. (1988). Realidad mental y mundos posibles. Barcelona, España: Gedisa.

Bruner, J. (1991). Actos de significado: Más allá de la revolución cognitiva. Madrid, España: Alianza Editorial. Cai, H., Brown, J. D., Deng, C. y Oakes, M. A. (2007). Self-esteem and culture: Differences in cognitive selfevaluations or affective self-regard? Asian Journal of Social Psychology, 10(3), 162-170. https://doi. org/10.1111/j.1467-839X.2007.00222.X

Casari, L., Albanesi, S. y Maristany, M. P. (2013). Influencia del enfoque teórico en el estilo personal del terapeuta. Psicogente, 16(29), 132-142. Recuperado de: http://revistas.unisimon.edu.co/index.php/psicogente/ article/view/1946 
Casari, L., Albanesi, S., Maristany, M. y Ison, M. (2016). Estilo Personal del Terapeuta en Psicoterapeutas de Adicciones. Revista Argentina de Clínica Psicológica, 25(1), 17-26. Recuperado de: https://www.redalyc. org/pdf/2819/281946989003.pdf

Castañeiras, C., Ledesma, R, García, F. y Fernández-Álvarez, H. (2008). Evaluación del Estilo Personal del Terapeuta: presentación de una versión abreviada del Cuestionario EPT-C. Terapia Psicológica, 26(1), 5-13. https://doi.org/10.4067/S0718-48082008000100001

Cimbolli, P., Quiñones, A., Ugarte, C. y Ceric, F. (2017). ADAPTACIÓN DEL EPT-C en Italia. Manuscrito no publicado.

Da Silva, M., Tellet, M., Fernández-Álvarez, H. y García, F. (2006). Estilo pessoal do terapeuta: Dados preliminares da versão brasileira do EPT-Q. Revista Psico, 37(3), 241-247. Recuperado de: https:/core.ac.uk/ download/pdf/25531788.pdf

Fernández-Álvarez, H. y García, F. (1998). El Estilo Personal del Terapeuta: Inventario para su evaluación. En S. Gril, A. Ibáñez, I. Mosca y P. L. R. Sousa (Eds.), Investigación en Psicoterapia (pp.76-84). Pelotas, Brasil: Educat.

Fernández Álvarez, H. y Gracía, F. (2019). El estilo personal del terapeuta. Buenos Aires, Argentina: Polemos.

Fernández-Álvarez, H., García, F. y Schreb, E. (1998). The research program at AIGLE. Journal of Clinical Psychology, 54(3), 343-359. https://doi.org/10.1002/(SICI)1097-4679(199804)54:3\%3C343::AIDJCLP5\%3E3.0.CO;2-Q

Fernández-Álvarez, H., García, F., Lo Bianco, J. y Corbella, S. (2000, septiembre). Estilos personales en terapeutas psicoanalíticos, cognitivos e integrativos. Trabajo presentado en el II Congresso Latinoamericano de Pesquisa em Psicoanalise e Psicoterapia, Gramado, Brasil.

Fernández-Álvarez, H., García, F., Lo Bianco, J. y Corbella, S. (2003). Assesment Questionnaire on The Personal Style of the Therapist PST-Q. Clinical Psychology and Psychotherapy, 10(2), 116-125. https://doi. org/10.1002/cpp.358

García, F., Castañeiras, C. y Fernández Álvarez, H. (2008). La investigación en la clínica. En H. Fernández Álvarez (comp.), Integración y Salud Mental (pp. 139-175). Bilbao, España: Desclée de Brouwer.

García, F. y Fernández-Álvarez, H. (2007). Investigación empírica sobre el Estilo Personal del Terapeuta: Una actualización. Revista Argentina de Clínica Psicológica, 14(2), 121-128. Recuperado de: http://repositorio. ub.edu.ar/handle/123456789/2940

García, F., Castañeiras, C, Rial, V. y Fernández-Álvarez, H. (2005). Programa de investigación sobre el estilo personal del terapeuta. Sesión de póster presentado en en el IV World Congress of Psychotherapy. Buenos Aires, Argentina.

La Roche, M. J. y Christopher, M. S. (2009). Changing paradigms from empirically supported treatment to evidence-based practice: A cultural perspective. Professional Psychology: Research and Practice, 40(4), 396-402. https://doi.org/10.1037/a0015240

Lee, J. A., Neimeyer, G. J. y Rice, K. G. (2013). The Relationship between Therapist Epistemology, Therapy Style, Working Alliance, and Interventions Use. American Journal of Psychotherapy, 67(4), 323-345. https://doi. org/10.1176/appi.psychotherapy.2013.67.4.323

Marsella, A. J. y Yamada, A. M. (2010). Culture and psychopathology: Foundations, issues, directions. Journal of Pacific Rim Psychology, 4(2), 103-115. https://doi.org/10.1375/prp.4.2.103

Orlinsky, D. E., Grawe, K. y Parks, B. K. (1994). Process and outcome in psychotherapy: Noch einmal. En A. E., Bergin y S. L., Garfield (Eds.), Handbook of psychotherapy and behavior change (pp. 270-376). Nueva York, NY: Wiley.

Orlinsky, D. E. y Howard, K. I. (1986). Process and outcome in psychotherapy. En S. L., Garfield y A. E., Bergin (Eds.), Handbook of psychotherapy and behavior change (pp. 311-381). Nueva York, NY: Wiley.

Prado-Abril, J., Fernández-Álvarez, J., Sánchez-Reales, S., Jeong Youn, S., Inchausti, F. y Molinari, G. (2019). La persona del terapeuta. Validación española del Cuestionario de Evaluación del Estilo Personal del Terapeuta (EPT-C). Revista de Psicopatología y Psicología Clínica, 24(3), 131-140. Recuperado de: http://revistas. uned.es/index.php/RPPC/article/view/24367/pdf

Quiñones, A. (2013). Indicadores de procesos en psicoterapia asociados a éxito. (Tesis Doctoral Universidad Autónoma de Barcelona, España). Recuperado de: https://ddd.uab.cat/record/118639

Quiñones, A., Ceric, F. y Ugarte, C. (2015). Flujos de información en zonas de tiempo subjetivo: estudio de un proceso psicoterapéutico exitoso. Revista Argentina de Clínica Psicológica, 24(3), 255-266. Recuperado: https://www.redalyc.org/pdf/2819/281946988007.pdf

Quiñones, A., Ceric, F., Ugarte, C. y De Pascale, A. (2017). Psychotherapy and psychological time: a case study. Rivista di Psichiatria, 52(3), 109-116. https://doi.org/10.1708/2722.27762 
Quiñones, A., Melipillán, R. y Ramírez, P. (2010). Estudio Psicométrico del Cuestionario de Evaluación del Estilo Personal del Terapeuta (EPT-C) en Psicoterapeutas Acreditados en Chile. Revista Argentina de Clínica Psicológica, 19(3), 273-281.

Quiñones, A., Melipillán, R. y Ugarte, C. (2012). Indicadores de procesos de éxito en psicoterapia cognitiva. Revista Argentina de Clínica Psicológica, 21(3), 247-254. Recuperado de: https://www.redalyc.org/ pdf/2819/281929021005.pdf

Quiñones, A. y Ugarte, C. (2019). Investigación del estilo personal del terapeuta post-racionalista: Una revisión [Research of the personal style of the post-rationalist therapist: A review]. Revista de Psicoterapia, 30(112), 7-15. https://doi.org/10.33898/rdp.v30i112.281

Quiñones, A., Ugarte, C., Ceric, F., Garcia, F. y Santibañez, P. (2019). Estilo personal del terapeuta: comparación entre terapeutas cognitivos post-racionalistas y sistémicos. Revista Argentina de Clínica Psicológica, 28(1), 48-55. https://doi.org/10.24205/03276716.2018.1054

Staczan, P., Schmuecker, R., Koehler, M., Berglar, J., Crameri, A., von Wyl, A., ... Tschuschke, V. (2017). Effects of sex and gender in ten types of psychotherapy. Psychotherapy Research, 27(1), 74-88. https://doi.org/ 10.1080/10503307.2015.1072285

Vázquez, L. y Güitiérrez de Vázquez, M. (2015). Orientación Teórico-Técnica y Estilo Personal del Terapeuta. Revista Argentina de Clínica Psicológica, 24(2), 133-142. Recuperado de: https://www.redalyc.org/ pdf/2819/281946783005.pdf

Vides-Porras, A., Grazioso, M. y García de la Cadena, C. (2012). El estilo personal del terapeuta guatemalteco en el interior del país. Revista Argentina de Clínica Psicológica, 21(1), 33-43. Recuperado de: https://www. redalyc.org/pdf/2819/281925884004.pdf

Wampold, B. E. (2015). How important are the common factors in psychotherapy? An update. World Psychiatry, 14(3), 270-277. https://doi.org/10.1002/wps.20238

Wampold, B. E. e Imel, Z. E. (2015). The great psychotherapy debate: The evidence for what makes psychotherapy work. Nueva York, NY: Routledge. https://doi.org/10.4324/9780203893340 